\title{
Programmed death ligand 1 expression in esophageal cancer following definitive chemoradiotherapy: Prognostic significance and association with inflammatory biomarkers
}

\author{
YATING TANG ${ }^{1}$, GUANG LI $^{1}$, SHAN WU $^{1}$, LINGRONG TANG ${ }^{1}$, \\ NING ZHANG ${ }^{2}$, JINZHAO LIU ${ }^{2}$, SHUO ZHANG ${ }^{1}$ and LEI YAO ${ }^{1}$ \\ Departments of ${ }^{1}$ Radiation Oncology and ${ }^{2}$ Pathology, The First Affiliated Hospital of China Medical University, \\ Shenyang, Liaoning 110001, P.R. China
}

Received October 17, 2016; Accepted January 4, 2018

DOI: $10.3892 / 01.2018 .7984$

\begin{abstract}
Immunotherapy with anti-programmed cell death protein 1 or programmed death ligand 1 (PD-L1) agents has demonstrated promising efficacy for the treatment of various types of malignancies. However, the role of PD-L1 as a tumor prognostic marker remains poorly understood. In the present study, the prognostic value of PD-L1 expression in esophageal carcinoma (EC) following definitive chemoradiotherapy (CRT) was investigated, and its associations with three systemic inflammation biomarkers, neutrophil-to-lymphocyte ratio (NLR), platelet-to-lymphocyte ratio (PLR) and lymphocyte-to-monocyte ratio (LMR) were further explored. A total of 104 patients with non-metastatic EC, who underwent definitive CRT between January 2009 and December 2012, were retrospectively analyzed. The expression of PD-L1 was examined by immunohistochemistry and the impact of PD-L1 expression level on overall survival (OS) was assessed. Furthermore, pretreatment neutrophil, lymphocyte, platelet and monocyte counts were obtained from routine blood tests to calculate the NLR, PLR and LMR. PD-L1 was overexpressed in EC compared with normal esophageal epithelium, with a positive expression rate of $37.5 \%$. Additionally, patients with positive PD-L1 expression had a lower NLR than those with negative PD-L1 expression $(\mathrm{P}=0.001)$. On multivariate analysis, the positive staining of PD-L1 was significantly associated with improved OS (HR, 0.6; 95\% CI, 0.372-0.965; $\mathrm{P}=0.035)$. Kaplan-Meier survival analysis showed a similar result $(\mathrm{P}=0.009)$. Additionally, sex (HR, 0.449; 95\% CI,
\end{abstract}

Correspondence to: Dr Guang Li, Department of Radiation Oncology, The First Affiliated Hospital of China Medical University, 155 North Nanjing Street, Heping, Shenyang, Liaoning 110001, P.R. China

E-mail: 13804058616@163.com; sy_liguang@163.com

Key words: esophageal cancer, programmed death ligand 1, neutrophil-lymphocyte ratio, platelet-lymphocyte ratio, lymphocyte-monocyte ratio, prognostic factors
0.229-0.880; $\mathrm{P}=0.020)$, clinical stage III (HR, 2.471; 95\% CI, 1.171-5.212; $\mathrm{P}=0.018)$, and receipt of concurrent chemoradiation (HR, 0.590; 95\% CI, 0.368-0.945; $\mathrm{P}=0.028$ ) were all independent prognostic factors in $\mathrm{EC}$ treated with definitive CRT. The correlation of NLR with PD-L1 expression validated the relevance of immunity and inflammation. In summary, the present study demonstrated that positive PD-L1 expression is associated with improved survival in patients with EC treated with radical CRT, indicating that PD-L1 is a promising prognostic marker.

\section{Introduction}

Esophageal cancer (EC) is one of the most aggressive types of cancers. In China, EC is the fourth leading cause of cancer-associated mortality (1). In Western countries, Barrett's adenocarcinoma is the most common histological type, whereas esophageal squamous cell carcinoma is dominant in East Asia (2,3). Among the currently available treatments, surgical resection is considered to be a potentially curative option for early stage EC. However, owing to the delay of diagnosis, the majority of patients will miss the optimal window for radical surgery. For patients with locally advanced $\mathrm{EC}$, radiotherapy combined with chemotherapy has become an important therapeutic strategy $(4,5)$. However, the prognosis of recurrent or metastatic EC remains poor, despite the development of novel chemotherapies and targeted drugs. Recently the roles of immune checkpoints and immunotherapies have been investigated in several types of malignancies $(6,7)$.

Programmed death-ligand 1 (PD-L1), is a 40-kDa transmembrane protein, and plays a major role in suppressing the immune system. The binding of PD-L1 to its receptor, PD-1, produces inhibitory signals, leading to evasion of the tumor from host monitoring and induction of therapeutic resistance $(8,9)$. PD-L1 has been reported to be highly expressed in various types of cancers and associated with tumor prognosis, such as in liver cancer, head and neck cancers, and lung cancer (10-14). Furthermore, numerous studies have found that PD-L1 overexpression could be considered a predictive biomarker for immune checkpoint inhibitors $(15,16)$. With regard to EC, although a number of studies have found that 
PD-L1 is overexpressed $(17,18)$, the predictive ability of PD-L1 remains controversial.

Increasing evidence has suggested that PD-L1 serves an anti-immune role through the regulation of inflammatory cytokines or signaling pathways. For example, the interleukin 6/Janus kinase/signal transducer and activator of transcription 3 (IL-6/JAK/STAT3) signaling pathway have been reported to regulate PD-L1 expression (19). The neutrophil-lymphocyte ratio (NLR), platelet-lymphocyte ratio (PLR) and lymphocyte-monocyte ratio (LMR), which are considered to reflect a systemic inflammatory response, have been reported to be associated with poor prognosis in various types of cancers (20-22). However, there is no consensus regarding the significance of these inflammatory parameters in the prognosis of EC. Furthermore, to date, no study has explored the association between PD-L1 and these systemic inflammatory markers in EC.

Based on these observations, a retrospective analysis of data was performed to assess the significance of PD-L1 expression for predicting survival outcomes in Asian patients treated with definitive CRT, and the associations between PD-L1 and inflammatory markers in EC were further investigated to provide novel insights into the development of immunotherapies.

\section{Materials and methods}

Patient eligibilityand tissue specimens. This study was approved by the Department of Radiation Oncology, The First Affiliated Hospital of China Medical University (Shenyang, China), and patients treated between January 2009 and December 2012 were included. A waiver of individual informed consent was granted. In this study, 104 patients were enrolled based on the following selection criteria: i) Confirmed EC by pathological diagnosis; ii) formalin-fixed paraffin-embedded (FFPE) specimens from pathological biopsy available; iii) all patients were treated with radical radiotherapy or definitive CRT initially and did not receive any prior treatments; iv) neutrophil, lymphocyte, platelet and monocyte counts could be obtained from medical records within a week prior to treatment. Upon application of these criteria, patients with inflammation and any malignancy with the exception of EC were excluded. Tumor stages were determined according to the seventh edition of American Joint Committee on Cancer/Union for International Cancer Control (AJCC/UICC) (23). In addition, 10 pairs of surgically resected cancer tissues were collected along with adjacent non-cancerous tissues as controls. Patient clinical and pathological information was also collected from medical records. Due to the restrictions of the selected conditions and the lack of necessary information, pretreatment complete blood profiles were only available for $83(79.8 \%)$ patients. Clinical characteristics are shown in Table I. Descriptive data are represented as means and standard deviations. The follow-up was conducted every 3 months for the first 2 years, then once every 6 months via personal interview or by telephone, the deadline was July 2016.

Treatment. A non-invasive mask was used to immobilize the patient's head and neck during treatment. The primary esophageal gross tumor volumes (GTV-nx) together with involved metastatic lymph nodes (GTV-nd) were determined from imaging examinations, barium meal and endoscopic findings. Clinical target volume (CTV) was defined as the GTV plus 3-5 $\mathrm{mm}$ to the anterior, posterior, right and left directions and $2.5 \mathrm{~cm}$ into the superior and inferior regions. CTV also encompassed a supraclavicular lymphonodus drawing region (in patients with upper or middle EC) or the drainage area of the lymph nodes around the stomach and cardia (in patients with lower EC). A margin of $0.5 \mathrm{~cm}$ in all directions was added to the CTV to generate the planning target volume. The organs at risk were delineated, including the spinal cord, lung and heart/pericardium. CT-based treatment planning was performed using a Pinnacle radiation therapy planning system (version 9.0; Philips Medical systems B.V., Eindhoven, The Netherlands). Patients were treated with intensity-modulated radiation therapy or three-dimensional conformal radiotherapy, which was delivered by a PRIMUS ${ }^{\mathrm{TM}}$ linear accelerator (Siemens, AG, Munich, Germany) using 6-MV photon beams. The radiotherapy regimens were $2.0 \mathrm{~Gy} /$ day, five times weekly, with a total dose of 60 or $66 \mathrm{~Gy}$. In addition, among the 104 patients enrolled, 41 received concurrent chemotherapy with cisplatin $\left(75 \mathrm{mg} / \mathrm{m}^{2}\right)$ by intravenous injection (IV) on day 1 and 5 -flurouracil $\left(1,000 \mathrm{mg} / \mathrm{m}^{2}\right)$ continuous IV on days 1-4; starting on the first day of irradiation and repeated after 21 days, 10 received sequential chemotherapy with four cycles of chemotherapy before radiotherapy, using the same schedule, And 53 patients received radiotherapy alone.

Immunohistochemical staining (IHC). FFPE tumor tissues (4- $\mu \mathrm{m}$ thick) were subjected to IHC with a streptavidin-peroxidase (SP) method (Biotin-Streptavidin Immunohistochemistry Kit; cat no. SP-9001; ZSGB-Bio, Beijing, China). The sections were de-waxed in xylene and ethanol. Antigen retrieval was performed by using a pressure cooker to heat tissue sections in Tris-ethylene-diamine tetra-acetic acid buffer $(\mathrm{pH} 9.0)$ for $\sim 4$ min. Endogenous peroxidase activity was blocked with $0.3 \% \mathrm{H}_{2} \mathrm{O}_{2}$ for 20 min followed by washing twice in phosphate-buffered saline (PBS). The sections were mounted on glass slides, pre-incubated with the blocking serum from the kit (liquid A) and incubated at $4{ }^{\circ} \mathrm{C}$ overnight with a monoclonal anti-PD-L1 antibody at a 1:300 dilution (cat. no. ab205921; Abcam, Cambridge UK) or PBS instead of primary antibodies as a blank control. Subsequently, incubation at $25^{\circ} \mathrm{C}$ for $10 \mathrm{~min}$ with the biotinylated secondary antibody from the kit (liquid B), horseradish peroxidase $\left(25^{\circ} \mathrm{C}, 15 \mathrm{~min}\right.$, liquid C) and 3,3'-diaminobenzidine chromogen (ZSGB-Bio) were performed sequentially. The slides were then counterstained with hematoxylin. Following dehydration with xylene and gradient ethanol (concentrations, 70, 75, 80, 90, 95 and 100\%, in turn) the sections were covered with neutral balsam.

Evaluation of PD-L1 expression. Immunohistochemical slides were observed under low-power magnification (x40) to identify the extent of staining, and immunostaining was further evaluated at high-power magnification (x200). Two independent observers blinded to all of the clinical data assessed PD-L1 expression semi-quantitatively. PD-L1 expression-positive cases were determined by staining intensity and the percentage of positive tumor cells, according to a method described previously (24). Staining intensity was scored on a 4-point scale: 0 , no staining; 1 , weak staining; 2 , moderate 
staining; and 3, strong staining respectively. The proportion of positive cells were scored as follows: 0,0\% stained cells; $1,1-30 \%$ stained cells; $2,30-60 \%$ stained cells; and 3, >60\% stained cells. Two observers discussed controversial cases, and a single consensus score was established by multiplying the scores for intensity and extent of staining. A receiver-operating characteristic (ROC) curve was calculated and the appropriate cut-off value was selected to distinguish between negative and positive cases. A score of $\geq 2$ was considered to represent positive expression of PD-L1.

Statistical analysis. All statistical analyses were conducted by SPSS 16.0 software. (SPSS, Inc., Chicago, IL, USA) The associations between the clinicopathological factors and PD-L1 expression were explored using a $\chi^{2}$ test. The values of NLR, PLR and LMR were compared according to PD-L1 expression status (positive/negative) using a Student's t-test. Spearman's correlation tests were used to analyze possible associations. Cox proportional hazards models with univariate and multivariate analyses were performed to assess the associations of clinicopathological factors with OS. The OS rates were calculated using the Kaplan-Meier method and were compared using the log-rank test. All tests were two-sided and $\mathrm{P}<0.05$ was considered to indicate a statistically significant difference.

\section{Results}

$P D-L 1$ expression in EC. The clinicopathological characteristics of the 104 patients with EC are presented in Table I. Fig. 1A indicated that there was no PD-L1 protein staining in normal esophageal epithelium (magnification x200). In Fig. 1B, the negative expression in EC was demonstrated (magnification $\mathrm{x} 200$ ), as shown in Fig. 1C and D, PD-L1 was overexpressed on the membrane or in the cytoplasm (or both) of EC cells under different magnifications (x100 and x200, respectively) in some cases, in contrast to normal esophageal epithelial tissues. Of the 104 EC tissues, 39 (37.5\%) showed positive PD-L1 staining and focal distribution. No statistically significant differences in clinical parameters were noted between the groups of positive and negative PD-L1 expression (Table II).

Association between PD-L1 expression and systemic inflammation biomarkers. Among the 83 patients for whom the complete pretreatment blood profiles were available, the mean values of NLR, PLR and LMR were 2.64 $\pm 1.34,138.87 \pm 64.69$ and $4.62 \pm 2.30$, respectively (Table I). To further investigate the potential correlation of these blood biomarkers with PD-L1 expression in EC tissues, the 83 patients were divided into two groups according to PD-L1 expression level. The distributions of these blood parameters are shown in the box diagrams (Fig. 2). The only statistically significant association identified was between NLR and PD-L1 expression level (Student's t-test, $\mathrm{P}=0.001$ ); patients in the PD-L1(+) group had lower NLRs than those in the PD-L1(-) group (Spearman correlation, $\mathrm{r}=-0.308$; $\mathrm{P}=0.005)$.

Prognostic significance of clinical factors and PD-L1 expression. On univariate analyses, it was identified that sex (female vs. male, $\mathrm{P}=0.029)$, T-classification (T3-4 vs. T1-2,
Table I. General clinical characteristics of the 104 patients.

\begin{tabular}{|c|c|}
\hline Variables & Value \\
\hline \multicolumn{2}{|c|}{ Age (years) } \\
\hline$\leq 65$ & $53(51.0)$ \\
\hline$>65$ & $51(49.0)$ \\
\hline \multicolumn{2}{|l|}{ Sex } \\
\hline Male & $88(84.6)$ \\
\hline Female & $16(15.4)$ \\
\hline \multicolumn{2}{|c|}{ Pathological type } \\
\hline SCC & $99(95.2)$ \\
\hline Others & $5(4.8)$ \\
\hline \multicolumn{2}{|l|}{ Location } \\
\hline Upper & $29(27.9)$ \\
\hline Middle & $52(50.0)$ \\
\hline Lower & $23(22.1)$ \\
\hline \multicolumn{2}{|c|}{ Length (cm) } \\
\hline$\leq 5$ & $46(44.2)$ \\
\hline$>5-7$ & $26(25.0)$ \\
\hline$>7$ & $32(30.8)$ \\
\hline \multicolumn{2}{|c|}{ T-classification } \\
\hline $\mathrm{T} 1-2$ & $14(13.5)$ \\
\hline $\mathrm{T} 3-4$ & $90(86.5)$ \\
\hline \multicolumn{2}{|c|}{ N-classification } \\
\hline No & $36(34.6)$ \\
\hline N1 & $63(60.6)$ \\
\hline $\mathrm{N} 2$ & $5(4.8)$ \\
\hline \multicolumn{2}{|c|}{ Clinic stage } \\
\hline I & $5(4.8)$ \\
\hline II & $21(20.2)$ \\
\hline III & $78(75.0)$ \\
\hline
\end{tabular}

Radiotherapy dose (Gy)

60

66

Therapeutic method

Radiotherapy alone

$53(51.0)$

Concurrent chemoradiation

$41(39.4)$

Sequential chemoradiation

Neutrophil count $\left(\mathrm{x} 10^{9} / \mathrm{ml}\right)$

$4.73 \pm 1.89$

Lymphocyte count (x10 $/ \mathrm{ml})$

$2.00 \pm 0.71$

Platelet count $\left(\mathrm{x} 10^{9} / \mathrm{ml}\right)$

$244.58 \pm 78.27$

Monocyte count $\left(\mathrm{x} 10^{9} / \mathrm{ml}\right)$

$0.51 \pm 0.25$

NLR

$2.64 \pm 1.34$

PLR

$138.87 \pm 64.69$

LMR

$4.62 \pm 2.30$

All values are presented as the number of patients (with \%), except for neutrophil, lymphocyte, platelet and monocyte counts, and NLR, PLR and LMR, which are presented as the mean \pm standard deviation. SCC, squamous cell carcinoma; NLR, neutrophil-to-lymphocyte ratio; PLR, platelet-to-leukocyte ratio; LMR, lymphocyte-to-monocyte ratio. 
Table II. Association between PD-L1 expression and clinical parameters.

\begin{tabular}{|c|c|c|c|c|}
\hline \multirow[b]{2}{*}{ Variables } & \multirow[b]{2}{*}{ Total cases, $\mathrm{n}$} & \multicolumn{2}{|c|}{ PD-L1 expression, $\mathrm{n}$} & \multirow[b]{2}{*}{ P-value } \\
\hline & & Negative & Positive & \\
\hline Patients & 104 & 65 & 39 & \\
\hline Age (years) & & & & 0.447 \\
\hline$\leq 65$ & 53 & 35 & 18 & \\
\hline$>65$ & 51 & 30 & 21 & \\
\hline Sex & & & & 0.575 \\
\hline Male & 88 & 54 & 34 & \\
\hline Female & 16 & 11 & 5 & \\
\hline Pathological type & & & & 0.064 \\
\hline $\mathrm{SCC}$ & 99 & 64 & 35 & \\
\hline Others & 5 & 1 & 4 & \\
\hline Location & & & & 0.417 \\
\hline Upper & 29 & 21 & 8 & \\
\hline Middle & 52 & 31 & 21 & \\
\hline Lower & 23 & 13 & 10 & \\
\hline Length (cm) & & & & 0.273 \\
\hline$\leq 5$ & 46 & 25 & 21 & \\
\hline$>5-7$ & 26 & 17 & 9 & \\
\hline$>7$ & 32 & 23 & 9 & \\
\hline T-classification & & & & 0.103 \\
\hline T1-2 & 14 & 6 & 8 & \\
\hline T3-4 & 90 & 59 & 31 & \\
\hline N-classification & & & & 0.966 \\
\hline No & 36 & 22 & 14 & \\
\hline N1 & 63 & 40 & 23 & \\
\hline $\mathrm{N} 2$ & 5 & 3 & 2 & \\
\hline Clinical stage & & & & 0.276 \\
\hline I & 5 & 2 & 3 & \\
\hline II & 21 & 11 & 10 & \\
\hline III & 78 & 52 & 26 & \\
\hline
\end{tabular}

PD-L1, programmed death ligand 1; SCC, squamous cell carcinoma; T-, tumor; N-, node.

$\mathrm{P}=0.045$ ), clinical stage (III vs. I-II, $\mathrm{P}=0.003$ ), concurrent chemotherapy (performed vs. not performed, $\mathrm{P}=0.028$ ) and PD-L1 expression (positive vs. negative, $\mathrm{P}=0.012$ ) were statistically significantly associated with OS (Table III). On subsequent multivariate analyses, sex (female vs. male; HR, 0.449; 95\% CI, 0.229-0.880; $\mathrm{P}=0.02$ ), clinical stage (III vs. I-II; HR, 2.471; 95\% CI, 1.171-5.212; $\mathrm{P}=0.018)$, concurrent chemotherapy (performed vs. not performed; HR, 0.590; 95\% CI, 0.368-0.945; $\mathrm{P}=0.028$ ) and PD-L1 expression (positive vs. negative; HR, 0.600; 95\% CI, 0.372-0.965; $\mathrm{P}=0.035$ ) were independent prognostic factors for patients with EC treated with radical CRT (Table III). Survival curves of these prognostic factors are shown in Fig. 3. The survival curve of PD-L1 demonstrated that patients with positive PD-L1 expression had increased survival times compared with patients with negative PD-L1 expression, and the median OS times were 26 and 11 months, respectively.

\section{Discussion}

The present study, investigated PD-L1 expression, the associations between PD-L1 expression and various inflammatory markers, and the prognostic relevance of these factors in patients with EC treated with definitive CRT. In contrast to previous studies $(9,17)$, this present study offered several novel observations that should be considered. The research on PD-L1 expression in EC was limited and controversial $(18,24,25)$. In the present patient cohort, $37.5 \%$ of EC tissue specimens exhibited positive staining. A review of previous studies regarding PD-L1 expression in EC was conducted (Table IV); the electronic databases PubMed up to September 2016 were systematically searched. The search was performed using the following terms: 'esophageal cancer;' 'programmed death ligand 1;' (or 'PD-L1'), 'prognosis.' In previous studies, the percentage of cases exhibiting positive PD-L1 expression 
A

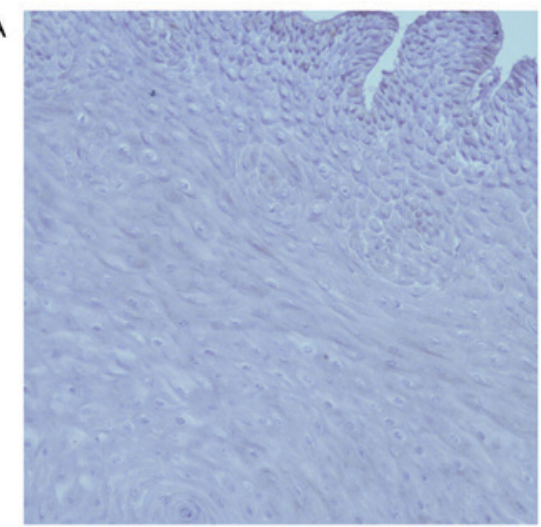

C

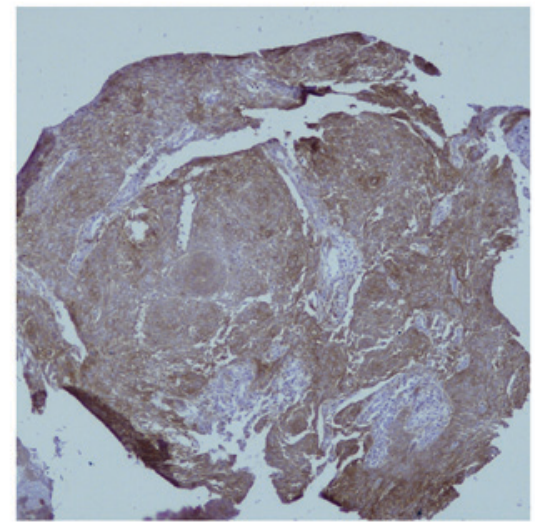

B

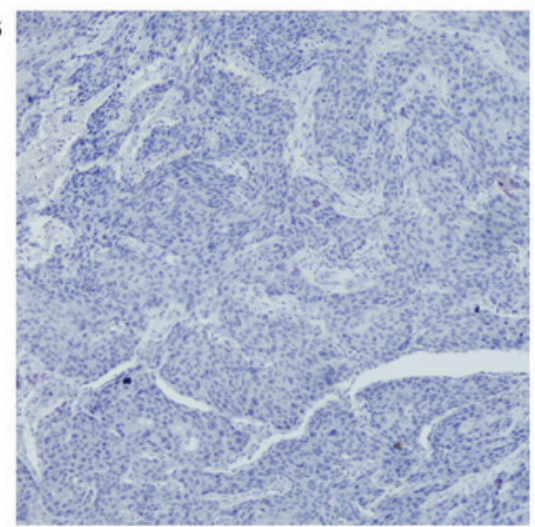

D

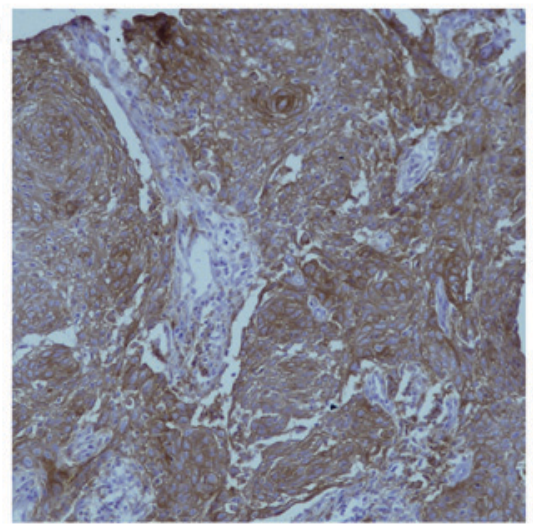

Figure 1. PD-L1 expression in EC and normal esophageal epithelium. (A) No PD-L1 staining in normal esophageal epithelium (magnification, x200). (B) Negative expression of PD-L1 in EC (magnification, x200). (C) Positive expression of PD-L1 in the cytoplasm and membrane of EC cells (magnification, $\mathrm{x} 100$ ). (D) Positive expression of PD-L1 in the cytoplasm and membrane of EC cells (magnification, x200). PD-L1, programmed death ligand 1; EC, esophageal carcinoma.
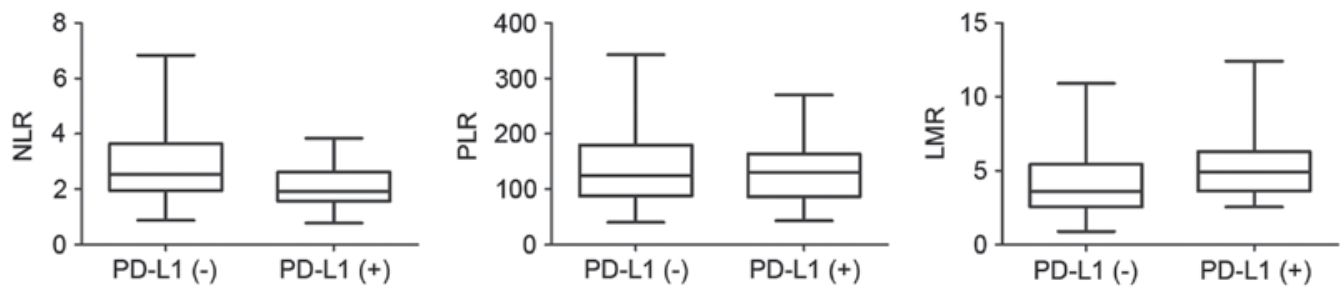

Figure 2. Correlation between PD-L1 and NLR, LMR and PLR. The two groups, divided according to the positive or negative expression of PD-L1, exhibited differences in NLR values ( $\mathrm{P}=0.001$, Student's $t$-test), whereas $\mathrm{PLR}$ and LMR did not differ significantly between the groups $(\mathrm{P}=0.448$ and $\mathrm{P}=0.056$, respectively). PD-L1, programmed death ligand 1; NLR, neutrophil-lymphocyte ratio; PLR, platelet-lymphocyte ratio; LMR; lymphocyte-monocyte ratio.

ranged from 18.4-82.8\%. Regarding the differences in PD-L1 expression rate, besides differences in the antibodies used, we speculate that differing PD-L1 evaluation criteria may be responsible for the large range. In the majority of studies, PD-L1 expression scores were determined according to the product of the percentage of stained tumor cells and the intensity of staining, and an appropriate cut-off value was selected to distinguish between negative and positive cases. However, in several reports on PD-L1 expression, the criterion for positive staining was $5 \%$ of tumor cells showing membrane staining. For instance, a cut-off value of 5\% was commonly used in lung cancer, renal cell carcinoma and melanoma (26-28). We speculate that different tumors may require different evaluation criteria due to tissue specificity. On the other hand, this divergence is from the PD-L1 itself. PD-L1 expression is dynamic, susceptible to the tumor microenvironment and unevenly distributed in tumor tissue. Thus, it is necessary to formulate a unified standard for evaluation and to explore more accurate detection methods in the future.

It is known that there is a complex relationship between inflammation and immunity, which together constitute the tumor microenvironment, and both are associated with invasion and recurrence in cancer patients. NLR, PLR and LMR are considered systemic inflammatory indicators and have been investigated in patients with various types of tumors (29-32); however, no consensus has been reached on their association with the prognosis of such patients. The present study explored the hypothesis that the blood parameters of NLR, PLR and LMR are associated with PD-L1 expression and OS. However, the results demonstrated no correlations between OS and NLR, PLR or LMR. Further studies utilizing a larger number of patients would be 
Table III. Univariate and multivariate analyses of risk factors associated with overall survival in patients treated with radical CRT.

\begin{tabular}{|c|c|c|c|c|c|c|c|c|}
\hline \multirow[b]{2}{*}{ Variables } & \multirow[b]{2}{*}{ Test group } & \multirow[b]{2}{*}{ Reference group } & \multicolumn{3}{|c|}{ Univariate analysis } & \multicolumn{3}{|c|}{ Multivariate analysis } \\
\hline & & & HR & $95 \% \mathrm{CI}$ & P-value & HR & $95 \% \mathrm{CI}$ & P-value \\
\hline Age (years) & $\leq 65$ & $>65$ & 0.992 & $0.974-1.010$ & 0.375 & - & - & - \\
\hline Sex & Female & Male & 0.478 & $0.246-0.929$ & $0.029^{\mathrm{a}}$ & 0.449 & $0.229-0.880$ & $0.020^{\mathrm{a}}$ \\
\hline Pathological type & $\mathrm{SCC}$ & Others & 1.824 & $0.573-5.804$ & 0.309 & - & - & - \\
\hline Tumor location & Lower & Upper/middle & 0.802 & $0.465-1.384$ & 0.428 & - & - & - \\
\hline Tumor length $(\mathrm{cm})$ & $>7$ & $\leq 5 / 5-7$ & 1.236 & $0.771-1.982$ & 0.379 & - & - & - \\
\hline T-classification & $\mathrm{T} 3-4$ & $\mathrm{~T} 1-2$ & 2.115 & $1.015-4.405$ & $0.045^{\mathrm{a}}$ & 0.833 & $0.309-2.247$ & 0.718 \\
\hline N-classification & $\mathrm{N}+$ & No & 1.354 & $0.852-2.152$ & 0.200 & - & - & - \\
\hline Clinical stage & III & $\mathrm{I} / \mathrm{II}$ & 2.326 & $1.341-4.035$ & $0.003^{\mathrm{a}}$ & 2.471 & $1.171-5.212$ & $0.018^{\mathrm{a}}$ \\
\hline Concurrent chemoradiation & Performed & Not performed & 0.602 & $0.383-0.948$ & $0.028^{\mathrm{a}}$ & 0.590 & $0.368-0.945$ & $0.028^{\mathrm{a}}$ \\
\hline PD-L1 expression & Positive & Negative & 0.552 & $0.348-0.877$ & $0.012^{\mathrm{a}}$ & 0.600 & $0.372-0.965$ & $0.035^{\mathrm{a}}$ \\
\hline NLR (mean) & $>2.64$ & $\leq 2.64$ & 1.310 & $0.796-2.156$ & 0.288 & - & - & - \\
\hline PLR (mean) & $>138.87$ & $\leq 138.87$ & 1.066 & $0.650-1.749$ & 0.800 & - & - & - \\
\hline LMR (mean) & $>4.62$ & $\leq 4.62$ & 0.687 & $0.420-1.123$ & 0.134 & - & - & - \\
\hline
\end{tabular}

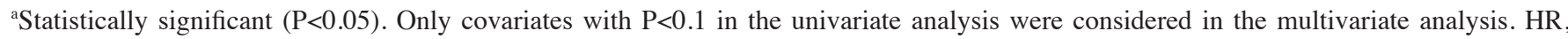
hazard ratio; CI, confidence interval; T-, tumor; N-, node; PD-L1, programmed death ligand 1; SCC, squamous cell carcinoma; NLR, neutrophil-to-lymphocyte ratio; PLR, platelet-to-leukocyte ratio; LMR, lymphocyte-to-monocyte ratio.
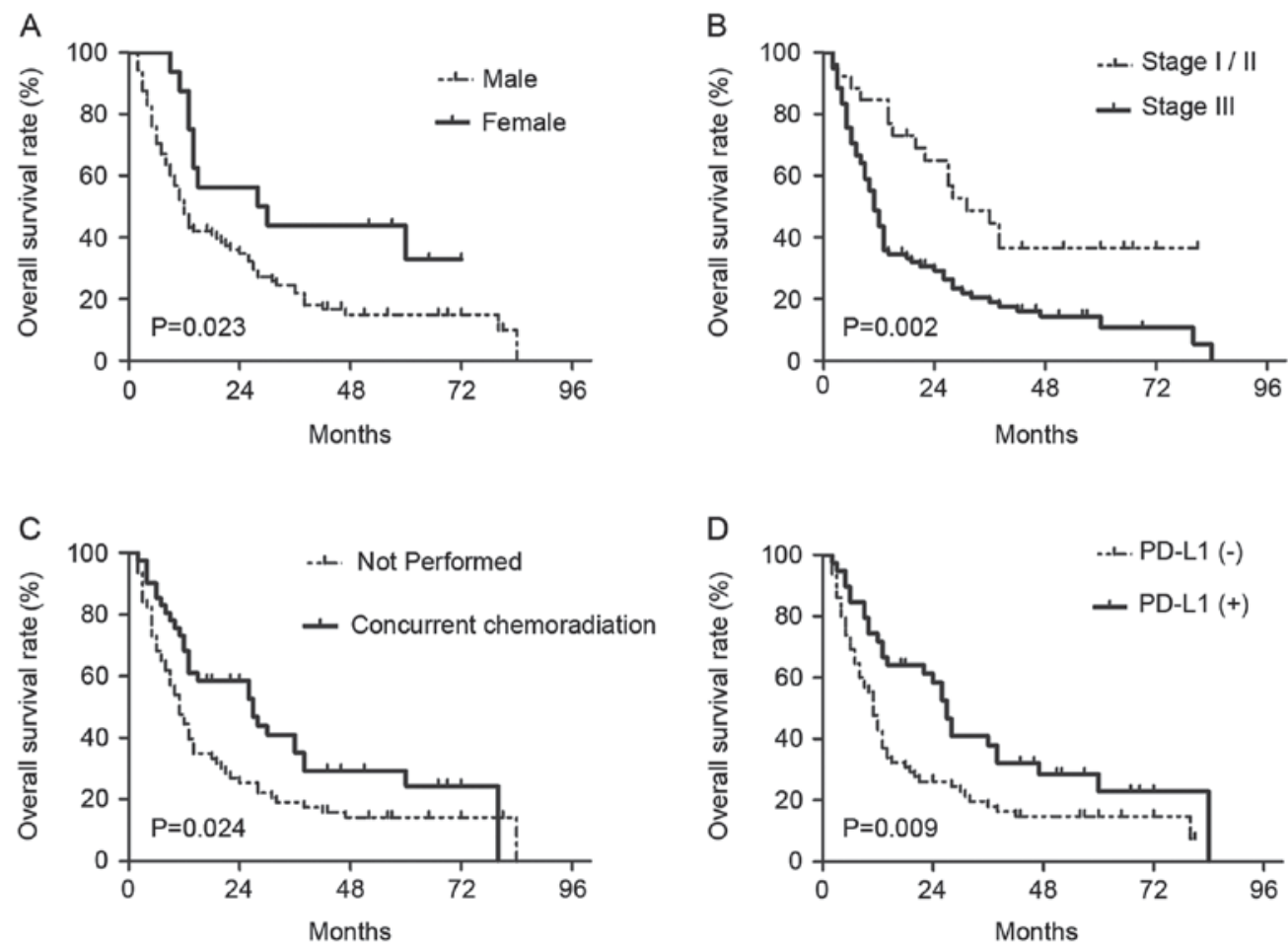

Figure 3. Kaplan-Meier curves showing differences in overall survival rates according to (A) sex, (B) clinical stage, (C) receipt of concurrent chemoradiation, and (D) PD-L1 expression in patients with esophageal carcinoma. P-values were determined by log-rank test. PD-L1, programmed death ligand 1.

beneficial. Notably, NLR was associated with PD-L1 expression, whereas PLR and LMR were not. To the best of our knowledge, the present study was the first to demonstrate the correlation between PD-L1 and systemic inflammatory markers in EC. Previous studies on the correlation between PD-L1 and NLR, PLR and LMR have only involved hepatocellular carcinoma (HCC). Wang et al (33) reported that, in hepatitis B-associated HCC, NLR was associated with PD-L1 expression within the center of the tumor, but NLR was not associated with PLR or prognostic nutritional index (PNI). Furthermore, the group with an NLR greater than the median had higher PD-L1 expression (33). In contrast to previous experiments, the present study showed that the NLR value was negatively correlated with PD-L1 expression. 


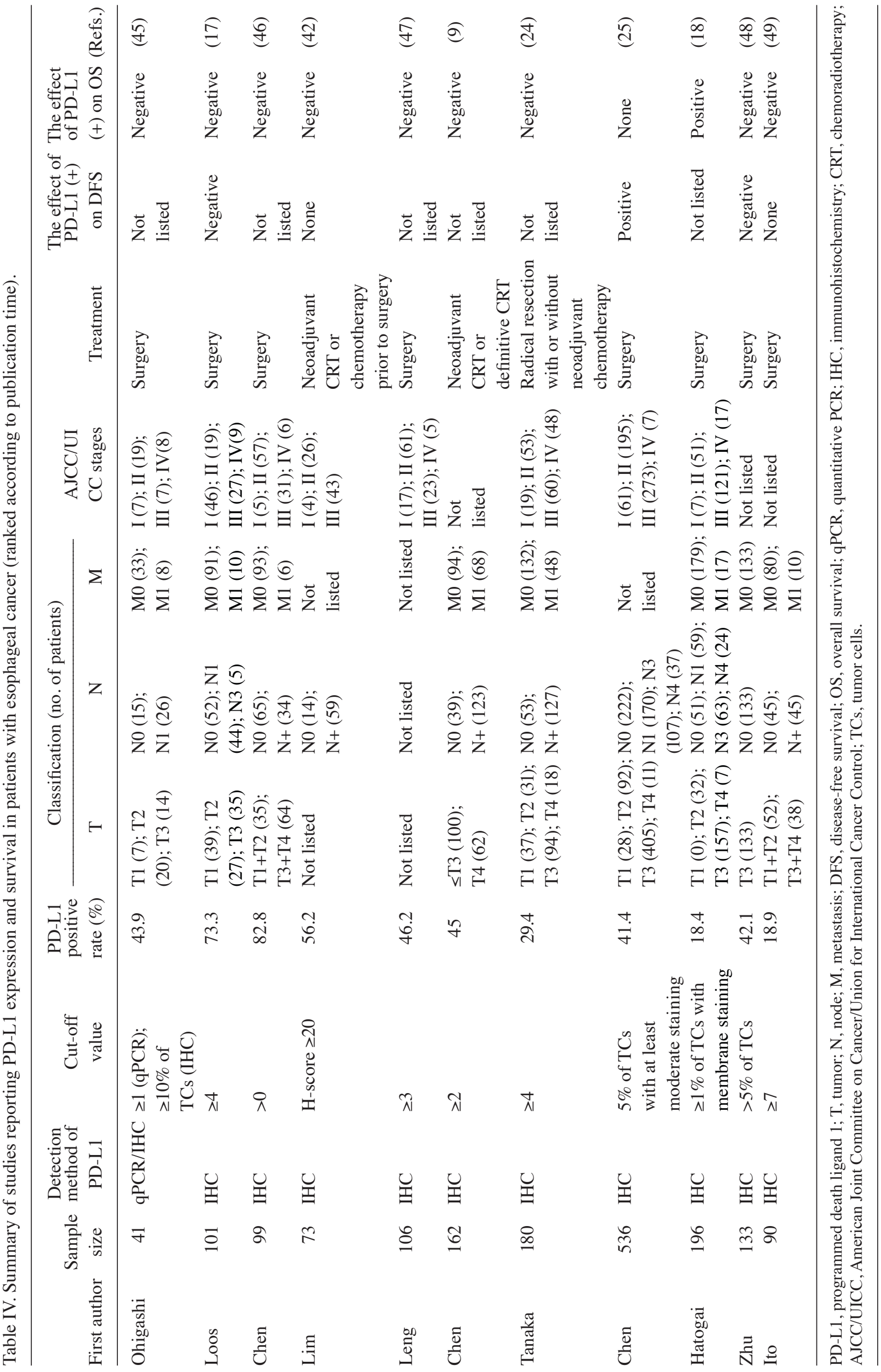


Cancer is a systemic disease; therefore, we considered the results that patients in the PD-L1 (+) group had lower NLRs than those in the PD-L1(-) group may be due to the host response to the tumor. It is reported that tumors are able to secrete a multitude of inflammatory factors, such as granulocyte-colony stimulating factor (G-CSF), IL-6, and interferon- $\gamma$ (IFN- $\gamma$ ), which could induce systemic reactions (34), and can affect the generation of blood cells. Furthermore, PD-L1 expression may be increased in response to cytokine exposure (such as G-CSF and IFN- $\gamma)(35,36)$. Thus, it is possible that the systemic inflammatory markers correlate with PD-L1 expression. In addition, T cells may act as an intermediary between PD-L1 and inflammatory markers. Several studies have suggested PD-L1 expression in human tumor tissue may lead to T cell exhaustion, while the pretreatment NLR level was found to be closely associated with $\mathrm{T}$ cell infiltration (37). Furthermore, the relationship between inflammation and immunity may change with the different stages of a tumor. Therefore, the mechanisms between PD-L1 and inflammation require further exploration. The aforementioned blood parameters possess the benefit of being easily obtained and low-cost. The relationship between NLR and PD-L1 expression may provide guidance for the development and adjustment of future immunotherapeutic strategies.

Previous studies have investigated the prognostic value of PD-L1 in EC (Table IV). Numerous reports demonstrated that patients with positive PD-L1 expression had a higher risk of mortality than patients lacking PD-L1 expression. However, in the current study, PD-L1 protein expression was a protective factor in patients treated with definitive CRT, consistent with the reports by Hatogai et al (18) and Chen et al (25) in surgical patients with EC. Furthermore, similar findings were reported in melanoma and, nasopharyngeal carcinoma as well as lung cancer $(28,38,39)$. It is well-established that the balance between cell birth and cell death is important; once this balance is disrupted, tumor progression will occur $(40,41)$. There may be other pathways or receptors associated with PD-L1 that have not yet been identified, which the effect of antitumor was stronger than the effect of PD-L1 leading to the evasion of tumor cells from host monitoring in tumor microenvironment, when PD-L1 is highly expressed. Furthermore, in all of the aforementioned studies, the patients received surgical intervention, whereas only patients who underwent definitive CRT were enrolled in the present study. This discrepancy between the current findings and previous findings may be explained by the different treatment strategies applied, as radiotherapy and chemotherapy may affect the expression of PD-L1 $(9,42)$. Additionally, radiation is able to produce an immunogenic effect in tumors, which may contribute to the host immune response against tumor cells $(43,44)$. Although a number of unanswered questions require further investigation, the current study suggested that the role of PD-L1 in the prognosis of patients treated with definitive CRT had significant value.

There are certain limitations to the current study. First, due to the retrospective nature, as well as the single-center design, certain data regarding immune parameters could not be obtained. Therefore, a prospective and multicenter study will be necessary in the future to confirm these results. In addition, the sample size was small, due to the restrictions of the inclusion and exclusion criteria. Finally, the, potential mechanisms linking PD-L1 expression with NLR, PLR and LMR were not investigated. Animal experiments and a large range of clinical studies will be conducted in due course.

In conclusion, these findings highlight that, in the tumor microenvironment, PD-L1 expression may reflect antitumor immunity and is a useful prognostic marker. The associations between PD-L1 expression and inflammatory markers provide a novel perspective for the further study of the mechanisms of inflammation and immunity. Given the complexity of the tumor microenvironment, a more comprehensive perspective on tumor therapeutic strategies should be formulated in the future.

\section{References}

1. Chen $\mathrm{W}$, Zheng R,Zeng H and Zhang S: The incidence and mortality of major cancers in China, 2012. Chin J Cancer 35: 73, 2016.

2. Parkin DM, Läärä E and Muir CS: Estimates of the worldwide frequency of sixteen major cancers in 1980. Int J Cancer 41: 184-197, 1988.

3. Ferlay J, Soerjomataram I, Dikshit R, Eser S, Mathers C, Rebelo M, Parkin DM, Forman D and Bray F: Cancer incidence and mortality worldwide: Sources, methods and major patterns in GLOBOCAN 2012. Int J Cancer 136: E359-E386, 2015.

4. Cooper JS, Guo MD, Herskovic A, Macdonald JS, Martenson JA Jr, Al-Sarraf M, Byhardt R, Russell AH, Beitler JJ, Spencer S, et al: Chemoradiotherapy of locally advanced esophageal cancer: Long-term follow-up of a prospective randomized trial (RTOG 85-01). Radiation therapy oncology group. JAMA 281: 1623-1627, 1999.

5. Ajani JA, Winter K, Komaki R, Kelsen DP, Minsky BD, Liao Z, Bradley J, Fromm M, Hornback D and Willett CG: Phase II randomized trial of two nonoperative regimens of induction chemotherapy followed by chemoradiation in patients with localized carcinoma of the esophagus: RTOG 0113. J Clin Oncol 26: 4551-4556, 2008.

6. Robert C, Schachter J, Long GV, Arance A, Grob JJ, Mortier L, Daud A, Carlino MS, McNeil C, Lotem M, et al: Pembrolizumab versus ipilimumab in advanced melanoma. N Engl J Med 372: 2521-2532, 2015.

7. Forde PM, Reiss KA, Zeidan AM and Brahmer JR: What lies within: Novel strategies in immunotherapy for non-small cell lung cancer. Oncologist 18: 1203-1213, 2013.

8. Ritprajak P and Azuma M: Intrinsic and extrinsic control of expression of the immunoregulatory molecule PD-L1 in epithelial cells and squamous cell carcinoma. Oral Oncol 51: 221-228, 2015.

9. Chen MF, Chen PT, Chen WC, Lu MS, Lin PY and Lee KD: The role of PD-L1 in the radiation response and prognosis for esophageal squamous cell carcinoma related to IL-6 and T-cell immunosuppression. Oncotarget 7: 7913-7924, 2016.

10. Brodská B, Otevřelová P and Kuželová K: Correlation of PD-L1 surface expression on leukemia cells with the ratio of PD-L1 mRNA variants and with electrophoretic mobility. Cancer Immunol Res 4: 815-819, 2016.

11. Gu X, Gao XS, Xiong W, Guo W, Han L, Bai Y, Peng C, Cui M and Xie M: Increased programmed death ligand-1 expression predicts poor prognosis in hepatocellular carcinoma patients. Onco Targets Ther 9: 4805-4813, 2016.

12. Inamura K, Yokouchi Y, Sakakibara R, Kobayashi M, Subat S, Ninomiya H, Nagano H, Nomura K, Okumura S and Ishikawa Y: Relationship of tumor PD-L1 expression with EGFR wild-type status and poor prognosis in lung adenocarcinoma. Jpn J Clin Oncol 46: 935-941, 2016.

13. Joneja U, Vranic S, Swensen J, Feldman R, Chen W, Kimbrough J, Xiao N, Reddy S, Palazzo J and Gatalica Z: Comprehensive profiling of metaplastic breast carcinomas reveals frequent overexpression of programmed death-ligand 1. J Clin Pathol 70: 255-259, 2017.

14. Zou MX, Peng AB, Lv GH, Wang XB , Li J, She XL and Jiang Y: Expression of programmed death-1 ligand (PD-L1) in tumor-infiltrating lymphocytes is associated with favorable spinal chordoma prognosis. Am J Transl Res 8: 3274-3287, 2016.

15. Aguiar P Jr, Lopes G, Santoro I, Tadokoro H, Barreto C and De Mello R: P2.47 (also presented as PD1.02): The role of PD-L1 expression as a predictive biomarker in advanced NSCLC: An update of a network meta-analysis: Track: Immunotherapy. J Thorac Oncol 11 (Suppl): S247-S248, 2016. 
16. Chen CL, Pan QZ, Zhao JJ, Wang Y, Li YQ, Wang QJ, Pan K, Weng DS, Jiang SS, Tang Y, et al: PD-L1 expression as a predictive biomarker for cytokine-induced killer cell immunotherapy in patients with hepatocellular carcinoma. Oncoimmunology 5: e1176653, 2016

17. Loos M, Langer R, Schuster T, Gertler R, Walch A, Rauser S, Friess H and Feith M: Clinical significance of the costimulatory molecule B7-H1 in Barrett carcinoma. Ann Thorac Surg 91: 1025-1031, 2011.

18. Hatogai K, Kitano S, Fujii S, Kojima T, Daiko H, Nomura S, Yoshino T, Ohtsu A, Takiguchi Y, Doi T and Ochiai A: Comprehensive immunohistochemical analysis of tumor microenvironment immune status in esophageal squamous cell carcinoma. Oncotarget 7: 47252-47264, 2016.

19. Zhang N, Zeng Y, Du W, Zhu J, Shen D, Liu Z and Huang JA: The EGFR pathway is involved in the regulation of PD-L1 expression via the IL-6/JAK/STAT3 signaling pathway in EGFR-mutated non-small cell lung cancer. Int J Oncol 49: 1360-1368, 2016.

20. Chen PC and Feng JF: A novel inflammation-based stage (I Stage) in patients with resectable esophageal squamous cell carcinoma. Mediators Inflamm 2016: 5396747, 2016.

21. Kara M, Uysal S, Altinisik U, Cevizci S, Güçlü $O$ and Dereköy FS: The pre-treatment neutrophil-to-lymphocyte ratio, platelet-to-lymphocyte ratio, and red cell distribution width predict prognosis in patients with laryngeal carcinoma. Eur Arch Otorhinolaryngol 274: 535-542, 2017.

22. van Kessel KE, de Haan LM, Fransen van de Putte EE, van Rhijn BW, de Wit R, van der Heijden MS, Zwarthoff EC and Boormans JL: Elevated derived neutrophil-to-lymphocyte ratio corresponds with poor outcome in patients undergoing pre-operative chemotherapy in muscle-invasive bladder cancer. Bladder Cancer 2: 351-360, 2016.

23. Edge SB and Compton CC: The American joint committee on cancer: The 7th edition of the AJCC cancer staging manual and the future of TNM. Ann Surg Oncol 17: 1471-1474, 2010.

24. Tanaka K, Miyata H, Sugimura K, Kanemura T, Hamada-Uematsu M, Mizote Y, Yamasaki M, Wada $\mathrm{H}$ Nakajima K, Takiguchi S, et al: Negative influence of programmed death-1-ligands on the survival of esophageal cancer patients treated with chemotherapy. Cancer Sci 107: 726-733, 2016.

25. Chen K, Cheng G, Zhang F, Zhang N, Li D, Jin J, Wu J, Ying L, Mao W and Su D: Prognostic significance of programmed death-1 and programmed death-ligand 1 expression in patients with esophageal squamous cell carcinoma. Oncotarget 7: 30772-30780, 2016

26. Takada K, Toyokawa G, Okamoto T, Akamine T, Takamori S, Katsura M, Fujishita T, Shoji F, Oda Y and Maehara Y: An immunohistochemical analysis of PD-L1 protein expression in surgically resected small cell lung cancer using different antibodies and criteria. Anticancer Res 36: 3409-3412, 2016.

27. Motzer RJ, Escudier B, McDermott DF, George S, Hammers HJ, Srinivas S, Tykodi SS, Sosman JA, Procopio G, Plimack ER, et al: Nivolumab versus everolimus in advanced renal-cell carcinoma. N Engl J Med 373: 1803-1813, 2015.

28. Taube JM, Anders RA, Young GD, Xu H, Sharma R, McMiller TL, Chen S, Klein AP, Pardoll DM, Topalian SL and Chen L: Colocalization of inflammatory response with B7-h1 expression in human melanocytic lesions supports an adaptive resistance mechanism of immune escape. Sci Transl Med 4: 127ra37, 2012.

29. Grimm M, Rieth J, Hoefert S, Krimmel M, Rieth S, Teriete P, Kluba S, Biegner T, Munz A and Reinert S: Standardized pretreatment inflammatory laboratory markers and calculated ratios in patients with oral squamous cell carcinoma. Eur Arch Otorhinolaryngol 273: 3371-3384, 2016.

30. Lee SM, Russell A and Hellawell G: Predictive value of pretreatment inflammation-based prognostic scores (neutrophil-to-lymphocyte ratio, platelet-to-lymphocyte ratio, and lymphocyte-to-monocyte ratio) for invasive bladder carcinoma. Korean J Urol 56: 749-755, 2015.

31. Liu JS, Huang Y, Yang X and Feng JF: A nomogram to predict prognostic values of various inflammatory biomarkers in patients with esophageal squamous cell carcinoma. Am J Cancer Res 5: 2180-2189, 2015

32. Stotz M, Liegl-Atzwanger B, Posch F, Mrsic E, Thalhammer M, Stojakovic T, Bezan A, Pichler M, Gerger A and Szkandera J: Blood-based biomarkers are associated with disease recurrence and survival in gastrointestinal stroma tumor patients after surgical resection. PLoS One 11: e0159448, 2016.

33. Wang Q, Blank S, Fiel MI, Kadri H, Luan W, Warren L, Zhu A, Deaderick PA, Sarpel U, Labow DM and Hiotis SP: The severity of liver fibrosis influences the prognostic value of inflammation-based scores in hepatitis B-associated hepatocellular carcinoma. Ann Surg Oncol 22 (Suppl 3): S1125-S1132, 2015.
34. He G, Zhang H, Zhou J, Wang B, Chen Y, Kong Y, Xie X, Wang X, Fei R, Wei L, et al: Peritumoural neutrophils negatively regulate adaptive immunity via the PD-L1/PD-1 signalling pathway in hepatocellular carcinoma. J Exp Clin Cancer Res 34: 141, 2015.

35. Bankey PE, Banerjee S, Zucchiatti A, De M, Sleem RW, Lin CF, Miller-Graziano CL and De AK: Cytokine induced expression of programmed death ligands in human neutrophils. Immunol Lett 129: 100-107, 2010

36. Thorn M, Guha P, Cunetta M, Espat NJ, Miller G, Junghans RP and Katz SC: Tumor-associated GM-CSF overexpression induces immunoinhibitory molecules via STAT3 in myeloid-suppressor cells infiltrating liver metastases. Cancer Gene Ther 23: 188-198, 2016.

37. Han S, Liu Y, Li Q, Li Z, Hou H and Wu A: Pre-treatment neutrophil-to-lymphocyte ratio is associated with neutrophil and T-cell infiltration and predicts clinical outcome in patients with glioblastoma. BMC Cancer 15: 617, 2015.

38. Lee VH, Lo AW, Leung CY, Shek WH, Kwong DL, Lam KO, Tong CC, Sze CK and Leung TW: Correlation of PD-L1 expression of tumor cells with survival outcomes after radical intensity-modulated radiation therapy for non-metastatic nasopharyngeal carcinoma. PLoS One 11: e0157969, 2016.

39. Song Z, Yu X and Zhang Y: Altered expression of programmed death-ligand 1 after neo-adjuvant chemotherapy in patients with lung squamous cell carcinoma. Lung Cancer 99: 166-171, 2016.

40. Wei Y, Huang H, Qiu Z, Li H, Tan J, Ren G and Wang X: NLRP1 overexpression is correlated with the tumorigenesis and proliferation of human breast tumor. Biomed Res Int 2017: 4938473, 2017.

41. Pang X, Li R, Shi D, Pan X, Ma C, Zhang G, Mu C and Chen W: Knockdown of Rhotekin 2 expression suppresses proliferation and induces apoptosis in colon cancer cells. Oncol Lett 14: 8028-8034, 2017.

42. Lim SH, Hong M, Ahn S, Choi YL, Kim KM, Oh D, Ahn YC, Jung SH, Ahn MJ, Park K, et al: Changes in tumour expression of programmed death-ligand 1 after neoadjuvant concurrent chemoradiotherapy in patients with squamous oesophageal cancer. Eur J Cancer 52: 1-9, 2016.

43. Demaria S, Golden EB and Formenti SC: Role of local radiation therapy in cancer immunotherapy. JAMA Oncol 1: 1325-1332, 2015.

44. Takeshima T, Pop LM, Laine A, Iyengar P, Vitetta ES and Hannan R: Key role for neutrophils in radiation-induced antitumor immune responses: Potentiation with G-CSF. Proc Natl Acad Sci USA 113: 11300-11305, 2016.

45. Ohigashi Y, Sho M, Yamada Y, Tsurui Y, Hamada K, Ikeda N, Mizuno T, Yoriki R, Kashizuka H, Yane K, et al: Clinical significance of programmed death-1 ligand-1 and programmed death-1 ligand-2 expression in human esophageal cancer. Clin Cancer Res 11: 2947-2953, 2005.

46. Chen L, Deng H, Lu M, Xu B, Wang Q, Jiang J and Wu C: B7-H1 expression associates with tumor invasion and predicts patient's survival in human esophageal cancer. Int J Clin Exp Pathol 7: 6015-6023, 2014.

47. Leng C, Li Y, Qin J, Ma J, Liu X, Cui Y, Sun H, Wang Z, Hua X, $\mathrm{Yu}$ Y, et al: Relationship between expression of PD-L1 and PD-L2 on esophageal squamous cell carcinoma and the antitumor effects of CD8+ T cells. Oncol Rep 35: 699-708, 2016.

48. Zhu Y, Li M, Mu D, Kong L, Zhang J, Zhao F, Li Z, Liu X, Bo C and $\mathrm{Yu}$ J: CD8+/FOXP3+ ratio and PD-L1 expression associated with survival in pT3N0M0 stage esophageal squamous cell cancer. Oncotarget 7: 71455-71465, 2016.

49. Ito S, Okano S, Morita M, Saeki H, Tsutsumi S, Tsukihara H, Nakashima Y, Ando K, Imamura Y, Ohgaki K, et al: Expression of PD-L1 and HLA class I in esophageal squamous cell carcinoma: Prognostic factors for patient outcome. Ann Surg Oncol 23 (Suppl 4): S508-S515, 2016

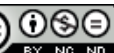

This work is licensed under a Creative Commons Attribution-NonCommercial-NoDerivatives 4.0 International (CC BY-NC-ND 4.0) License. 